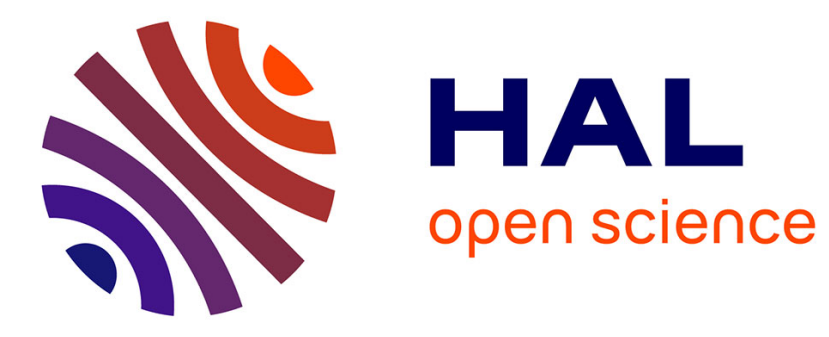

\title{
Reinforcement Learning for Interactive QoS-Aware Services Composition
}

\author{
Pegah Alizadeh, Aomar Osmani, Mohamed Essaid Khanouche, Abdelghani \\ Chibani, Yacine Y. Amirat
}

\section{- To cite this version:}

Pegah Alizadeh, Aomar Osmani, Mohamed Essaid Khanouche, Abdelghani Chibani, Yacine Y. Amirat. Reinforcement Learning for Interactive QoS-Aware Services Composition. IEEE Systems Journal, 2020，10.1109/JSYST.2020.2997069 . hal-02924667

\section{HAL Id: hal-02924667 https://hal.science/hal-02924667}

Submitted on 28 Aug 2020

HAL is a multi-disciplinary open access archive for the deposit and dissemination of scientific research documents, whether they are published or not. The documents may come from teaching and research institutions in France or abroad, or from public or private research centers.
L'archive ouverte pluridisciplinaire HAL, est destinée au dépôt et à la diffusion de documents scientifiques de niveau recherche, publiés ou non, émanant des établissements d'enseignement et de recherche français ou étrangers, des laboratoires publics ou privés. 


\title{
Reinforcement Learning for Interactive QoS-Aware Services Composition
}

\author{
Pegah Alizadeh ${ }^{\circledR}$, Aomar Osmani, Mohamed Essaid Khanouche ${ }^{(1)}$, Abdelghani Chibani, and Yacine Amirat ${ }^{(1)}$
}

\begin{abstract}
An important and challenging research problem in web of things is how to select an appropriate composition of concrete services in a dynamic and unpredictable environment. The main goal of this article is to select from all possible compositions the optimal one without knowing a priori the users' quality of service $(\mathrm{QoS})$ preferences. From a theoretical point of view, we give bounds on the problem search space. As the QoS user's preferences are unknown, we propose a vector-valued MDP approach for finding the optimal QoS-aware services composition. The algorithm alternatively solves MDP with dynamic programming and learns the preferences via direct queries to the user. An important feature of the proposed algorithm is that it is able to get the optimal composition and, at the same time, limits the number of interactions with the user. Experiments on a real-world large size dataset with more than 3500 web services show that our algorithm finds the optimal composite services with around 50 interactions with the user.
\end{abstract}

Index Terms-Quality of services (QoS), reinforcement learning (RL), services composition, web of things (WoT).

\section{INTRODUCTION}

$\mathbf{T}$ HE web of things (WoT) opens the way for the development of new intelligent applications that can be used to provide innovative and valuable services in several domains, such as smart cities, smart homes, ambient assisted living, and connected cars [11], [35]. One of the WoT challenges is to deal with services composition to ensure suitable services flexibility and customization. This is crucial to meet the evolving needs of users, which can be expressed through several parameters of quality of service (QoS) (such as response time, throughput, availability, price, popularity, etc.).

Following the web service paradigm [4] services, composition problems are specified as a workflow involving abstract and concrete services. A concrete service refers to an executable service, where an abstract service describes the abstract functionality of

Manuscript received October 25, 2019; revised April 15, 2020; accepted May 17, 2020. (Corresponding author: Pegah Alizadeh.)

Pegah Alizadeh is with the Léonard de Vinci Pôle Universitaire, Research Center, 92916 Paris La Défense, France (e-mail: pegah.alizadeh@ devinci.fr).

Aomar Osmani is with the LIPN, UMR CNRS 7030, Université Sorbonne Paris Nord, 93430 Villetaneuse, France (e-mail: ao@lipn.univ-paris13.fr).

Mohamed Essaid Khanouche is with the Medical Computing Laboratory, Faculty of Exact Sciences, University of Bejaia, Bejaia 06000, Algeria, and also with the University of Paris Est Creteil, LISSI, F-94400 Vitry, France (e-mail: mekhanouche@gmail.com).

Abdelghani Chibani and Yacine Amirat are with the University of Paris Est Creteil, LISSI, F-94400 Vitry, France (e-mail: chibani@u-pec.fr; amirat@ u-pec.fr).

Digital Object Identifier 10.1109/JSYST.2020.2997069 the concrete service. In environments such as the WoT, concrete services are web interfaces to objects functionalities, which are exposed by using functional and nonfunctional metadata. At a given run time and for a given user, each abstract service can be achieved using several concrete services with the same functionality but possibly with different nonfunctional parameters, such as the QoS. A composite service (workflow) specifies what are the concrete services that should be selected for each abstract service and what is the best services permutation to satisfy the user's requirements in terms of the QoS. An important and challenging research problem is, therefore, how to select an appropriate composition of concrete services in a dynamic and unpredictable environment [32].

Composing services to achieve more complex workflows can be formulated as a mathematical composition, which is always associative and commutative [15]. To limit the combinatorial explosion of the composition, specific domain constraints must be taken into account: same time execution for several concrete services is allowed, abstract services are used to instantiate unlimited number of concrete services and each concrete service appears at most once in the composition. Therefore, the services composition problem can be viewed as the selection of concrete services offering the overall best QoS.

Although state-of-the-art approaches find the services composition workflow by knowing the users' priorities in advance [20], [26], [36], these approaches are limited and static; therefore, they can not handle the following cases: 1) the presence of the user uncertainties before the service executions, 2) unpredictable services manners such as service failures. We propose a reinforcement learning (RL)-based approach to dynamically obtain the optimal service composition, according to the user's expectations on the QoSs. Markov Decision Processes (MDP) with unknown rewards enable us to tackle this challenge by optimizing rewards according to the answered queries for any user [1], [2]. Based on our approach and according to the given theoretical results, our algorithm minimizes the number of queries needed to select the best service composition. The proposed algorithm is validated with a large number of experiments on the most representative real-world dataset for our problem [50]. The experiments show that the proposed approach is able to provide the best composite service integrating dynamically user QoS preferences during the algorithm computation process. We also show some theoretical lower and upper bounds on the size of the search space of the composition problem.

The main contributions of this article are as follows. 
1) We propose the first approach in the literature able to provide a web services composition that explicitly takes into account the users' expectations and preferences on the QoS.

2) Within the given framework, we show a theoretical lower bound for the number of possible services compositions by respecting the mentioned constraints.

3) Our method is able to select an optimal services composition among all the possible permutations without knowing a priori the users' preferences.

4) We validate the algorithm on a real world dataset of web services.

The article is organized as follows: The next section summarizes the related work on QoS-aware services composition. Section III provides the problem formulation, together with the theoretical results on the search space. Section IV formalizes the services composition problem as an MDP. Section V details the proposed interactive RL algorithm to deal with the services composition problem and Section VI summarizes the experimental results. Finally, Section VII gives the conclusion and some perspectives.

\section{RELATED WORKS}

Usually, acquiring user preferences is not easy in practice, this is due to the fact that the users are not always certain about their preferences until the service execution. Moreover, collecting user preferences can be complex in terms of time and memory [33]. For these reasons, most of the services composition studies in the literature are based on the assumption of knowing the user preferences on the QoS attributes in advance.

For what concerns services composition problems where the user preferences are known in advance, several works are proposed in the literature [6], [14], [24], [36]. Such works can be classified accordingly to the techniques used: graph search [12], [31], Pareto optimality [44], [46], constraints decomposition [3], meta-heuristic population [7], [17], [39], [42], [49], planning [8], [51], integer linear programming [47], [48], recommender system [9], [22], [25], and machine learning [13], [26], [29], [30], [40]. In the following, we will describe with more details some of the mentioned papers.

A services selection algorithm combining qualitative and quantitative QoS attributes is proposed in [39]. The qualitative attributes considered in this approach are provider, location, and platform, whereas the quantitative attributes are response time, throughput, reliability, and availability. The services selection problem is solved using a global optimization algorithm and a genetic algorithm.

RL techniques have been often used to deal with dynamic and uncertain environments in services composition problems. In [30] and[40], the services composition problem in a dynamic environment is modeled as an MDP and solved using a Q-learning method. In these approaches, the QoS attribute values are extracted through executing the services, while the optimal workflow of elementary service invocation actions is updated according to the change occurred in the environment. In [40], Wang et al. used a normalized reward function combining the
QoS attributes and the known user preferences on the attributes, while the approached proposed in [30] aims at maximizing the expected cumulative rewards, which is on behalf of the satisfaction degree of the user's QoS constraints. In [26], two multiobjective MDP methods are proposed to handle services composition in uncertain and dynamic environments. The first approach addresses the single-policy multiobjective composition scenarios, whereas the second approach addresses the multiple-policies composition scenarios. In the first approach, the user's preferences are given a priori, whereas in the second one, the set of all suitable services compositions is represented as a convex hull of extreme services. Recently, several works have been proposed to use RL methods on large-scale services composition. For instance, Wang et al. [37] used hierarchical RL, Liu et al. [21], [38] utilized recurrent neural networks and deep RL approaches, and Yang and Xie[45] proposed an actor-critic method as a deep RL approach.

Many services composition approaches are based on machine learning technique different from RL. For instance, Hossain et al. [18], [23] proposed services selection approaches in the context of QoS-aware services composition. In [23], the services selection with global QoS constraints is first formulated as a set-based optimization problem, whereas the k-Means clustering method is then used to find the composite service by maximizing the QoS value and satisfying the global QoS constraints. On the other hand, Hossain et al. [18] presented an algorithm, which first uses the k-means clustering to obtain clusters of candidates services, then, for each cluster it obtains the composite service in terms of QoS using a heuristic method.

To the best of our knowledge, very few approaches have been proposed for solving the services composition problem in interaction with the users dynamically and without knowing the user's preferences a priori. The closest existing approaches are [9], [10], [20], [26], and [36]. In [9], Chen et al. proposed to extract the user preferences on the QoS attributes in advance, with a location-aware web service recommendation system. This system first collects users' QoS observations related to the past usage experience of different Web services. The users are then clustered, according to their locations and their observed QoS, in order to propose personalized service recommendations to each user. In [20], the services composition problem in highly dynamic environments is modeled as an uncertainly planning problem using a partially observable MDP. A time-based RL approach is then proposed to find the composite service satisfying the user's requirements. The proposed approach does not require knowledge of complete information about services. It uses historical information to estimate the success probability of a services' composition using results. Finally, Tsai et al. [36] suggested a user-centric service composition to identify the subset of correlated services that best match the users' requirements from a designed ontology.

Chen et al. [10], [26] introduced a method for finding the list of all nondominated workflows, regardless of the user preferences on the QoS attributes. Our approach goes beyond the identification of the nondominated workflows: it proposes an approach for identifying one optimal workflow (among the set of non-dominated workflows) for each system user. 
TABLE I

ENTRY From the REAL DATASET [49], [50] USED IN OUR EXPERIMENTS

\begin{tabular}{|c|c|c|c|c|c|}
\hline User & time & service & con. service & time resp. & throughput \\
\hline 97 & 5 & 19994 & 3104 & 0.238 & 0.773 \\
\hline
\end{tabular}

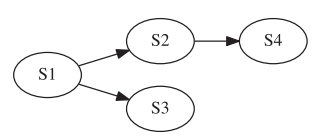

Fig. 1. Abstract services precedence graph of Example 2.

\section{PROBLEM Formulation}

For the sake of simplicity, the problem of identifying a services composition able to satisfy the end-to-end user requirements can be defined as follows. Given a set of $n$ abstract services $S=\left\{S_{1}, \ldots, S_{n}\right\}$ where each abstract service $S_{i}$ can be implemented by $n_{i}$ concrete services $\left\{S_{i 1}, \ldots, S_{i n_{i}}\right\}$. Each concrete service $S_{i j}$ may be executed by a set of actors $\left\{a_{1}, \ldots, a_{k}\right\}$. An actor can be an end-user or any other entity for which the service is rendered. We denote the concrete service $S_{i j}$ executed by the actor $a_{k}$, by $S_{i j}^{k}$. Furthermore, in several situations, the same service instance can be executed more than once over time by the same actor. In this work, we assume that the time horizon can be divided in time steps. The concrete service $S_{i j}$ executed by the actor $a_{k}$ at time $t$ is denoted by $S_{i j}^{k}(t)$ and is called execution. Example 1 shows a sample of a real case database for the services composition problem.

Example 1: Table I is an extracted line from the real dataset [49], [50] used in our experiments. According to the aforementioned notations, this line should be denoted as $S_{19994,3104}^{97}(5)$. It represents for the abstract service 19 994, the response time and throughput values of the concrete service 3104 executed by user 97 at time step 5 .

Each service performs functions that serve the actors. To evaluate the quality of a service at the application level, a set of criteria is used, such as response time, throughput, reliability, availability, price, etc. [43], [48].

In service-oriented architectures, the orchestration process composes the existing services in order to create a new service having central control over the whole process [19]. In our context, the main part of the orchestration process is the abstract service composition. This composition process is guided by one main constraint: an abstract service can have a set of prerequisite abstract services that must be executed in order to make its execution possible. All such precedence constraints can be represented with a suitable oriented graph (see Fig. 1). A path in the precedence graph represents a possible orchestration of abstract services.

We denote as orchestration of abstract services a sequence of subsets of the set of possible abstract services $S$, one for each time step (one abstract service can be included in at most one timestep). Moreover, we will consider only feasible abstract service orchestrations, i.e., orchestrations where an abstract service is included in a set at time steps $t$ only if all its prerequisite

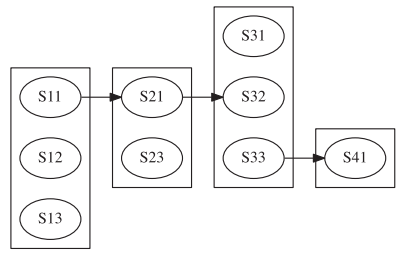

Fig. 2. Concrete workflow $\left(S_{11}, S_{21}, S_{32}, S_{41}\right)$ is a possible organization to realize the orchestration given in Fig. 1.

abstract services are included in the set of precedent time steps (see Example 2).

Example 2: Let $S_{1}, S_{2}, S_{3}$, and $S_{4}$ be four abstract services. Services $S_{2}$ and $S_{3}$ need outputs of the service $S_{1}$ and the service $S_{4}$ can be executed only after $S_{2}$. The abstract services precedence graph is given in Fig. 1. The possible orchestrations with respect to this graph are: $\left(S_{1},\left\{S_{2}, S_{3}\right\}, S_{4}\right),\left(S_{1}, S_{2}, S_{3}, S_{4}\right)$, $\left(S_{1}, S_{2}, S_{4}, S_{3}\right)$, etc.

Each abstract service may be realized by several concrete services. When an abstract service orchestration is defined, we need to choose an appropriate concrete service that performs each corresponding service. In the context of the WoT, services composition can be seen as an invocation workflow or plan of the services, which are exposed by the objects through the web. In this article, the possible concrete services organization for a given abstract service orchestration is represented by a concrete workflow. The concrete workflow must be coherent with the associated abstract service orchestration. Fig. 2 gives an example of a concrete workflow for the orchestration given in Fig. 1.

We define as workflow a concrete service concatenation of a given feasible abstract service orchestrations, i.e., a collection of subsets of concrete services obtained from a feasible abstract service orchestration after substituting each abstract service by one of its associated concrete services.

Let $M$ be the number of all possible feasible abstract service orchestrations and $\psi_{k}$ (for $k \in\{1, \ldots, M\}$ ) be the $k$ th possible orchestration. We are interested in defining an evaluation function $F\left(\psi_{k}\right)$ that associates a numerical value to all possible concrete service concatenations of $\psi_{k}$.

Let $\mathcal{Q}=\left\{q_{1}, \ldots, q_{m}\right\}$ be the set of all possible QoS criteria. The criteria can be applied both at the abstract and concrete levels. Without loss of generality, we limit the criteria to the concrete service level. In this case, $q_{l}\left(S_{i j}\right)$ denotes the quality value of the $q_{l}$ criteria for the $S_{i j}$ concrete service. We suppose that the values of all criteria are normalized.

To obtain $F\left(\psi_{k}\right)$, it is necessary to select the best concrete service concatenation, according to the set of criteria in $\mathcal{Q}$, among the concrete services in the given $\psi_{k}$ orchestration. In our context, the $F$ function is assumed to be a linear combination of these criteria (see Example 3).

Without loss of generality, the service selection problem can be defined as follows:

Find the orchestration $\psi_{k}$ with the best value of $F\left(\psi_{k}\right)$.

Example 3: Let us consider a problem with three abstract services $\left\{S_{1}, S_{2}, S_{3}\right\}$. Assume that this problem has a single 
possible orchestration $\psi_{1}=\left(S_{1}, S_{2}, S_{3}\right)$ such that $\psi_{1}$ represents a serial orchestration (we execute first concrete services of $S_{1}$, then $S_{2}$ and end with $S_{3}$ ). Let us consider a problem with two evaluation criteria $\mathcal{Q}=\left\{q_{1}, q_{2}\right\}$, for instance, throughput and service time response. To each abstract service $S_{i}$, we associate a value for $q_{i 1}$ and a value for $q_{i 2}$. The value of the orchestration is defined as a linear combination between these criteria: $\sum_{i=1}^{3}\left(w_{1} q_{i 1}+w_{2} q_{i 2}\right)$ where the coefficients $w_{i}$ (one for each abstract service $S_{i}$ ) are given by the actor (user).

From an operational point of view, the evaluation of the concrete services quality is estimated over their executions through the time. In the above example, the users' preferences are presented as weight values. In practice, the weights are not given a priori. In Section V, we propose an RL method based on MDPs for learning QoS attributes weights representing users' predefined preferences.

\section{A. On the Global Search Space Size}

The purpose of this section is to enumerate the number of end-to-end concrete service combinations (workflows) in order to evaluate the QoS of each combination and then to select the best one according to a given set of user/actor preferences. To the best of our knowledge, there is no existing work in the literature dealing with this enumeration problem. In the rest of the section, we give the size of the search space for some special cases and for the generic case, where no constraints are imposed on the structure of the orchestration. Those sizes can be viewed, respectively, as lower and upper bounds on the search space for any service combination problem.

Let us consider the generic case of a services composition problem with $n$ abstract services $S_{1}, \ldots, S_{n}$, such that each abstract service $S_{i}$ can be realized by exactly $m$ possible concrete services. In the following sections, we analyze how the size of the search space changes according to the different constraints on the service order and concurrency.

1) Total Order (sequential) Abstract Services: No explicit constraint on the order of execution of the services is imposed but at each time step, it is possible to execute only one abstract service. Since each $S_{i}$ can be realized by $m$ possible concrete services, for a given fixed ordering of abstract services, the possible number of realizations for each order is $m^{n}$. If there is no additional constraint between abstract services, the number of abstracts services permutations is $n$ !. In this case, the total number of workflows is $n ! m^{n}$.

2) Parallel Abstract Services: All abstract services are executed at the same time. In this case, there exists only one possible combination between abstract services. The only degree of freedom is to choose which concrete service to associate to each abstract service. For this reason, there exists $m^{n}$ possible realizations.

3) General Case: For each time step, it is possible to execute simultaneously any number (up to $n$ ) of abstract services. We recall that $S(n, k)$, the Stirling number of the second kind, counts the number of ways for partitioning an $n$-element set into $k$ subsets $^{1}$ [16]. On the contrary, $k$ subsets can be ordered in $k$ ! different ways. This leads to a total of $S(n, k) k$ ! different orchestrations of time length exactly $k$. This means that all abstract services in each set should be executed at the same time. Therefore, the total number of orchestrations is

$$
\sum_{k=1}^{n} S(n, k) k !=\sum_{k=1}^{n} \sum_{l=0}^{k}(-1)^{l}\left(\begin{array}{l}
k \\
l
\end{array}\right)(k-l)^{n} .
$$

For any given orchestration with $n$ abstract services and $m$ concrete services, we have $m^{n}$ total number of workflows. Notice that each abstract service is associated to one and only one executed concrete service at each time step. Consequently, to obtain the total number of workflows, we need to multiply the quantities given by (1) with the total number of workflows for each orchestration

$$
m^{n} \sum_{k=1}^{n} S(n, k) k !
$$

We finally notice that the situation is slightly different if we impose to associate the same concrete abstract to all the abstract services at the same time step. If this is the case, we have a total number of workflows equal to

$$
\sum_{k=1}^{n} S(n, k) k ! m^{k} .
$$

The results showed in this section give an idea of the combinatorial explosion one could expect if no precedence is imposed on the orchestrations. For this reason, we restrict this article to sequential orchestrations.

\section{SERVICES COMPOSITION AS AN MDP}

MDPs are often used to model sequential decision problems [27].

In this section, we describe how to solve the services composition problem via MDP models. We are looking for an optimal QoS-selection strategy satisfying the user's requirements in terms of QoS attributes. Since the user's preferences with respect to QoS attributes are unknown, we use a partially known MDP model, and more particularly, a vector-valued MDP (VMDP) model [2].

\section{A. Vector-Valued Markov Decision Process}

The following definitions are necessary to define our VMDP model.

Definition 1: A discrete-time MDP [1] is defined by a tuple $\left(T, S, A, P_{t}(. \mid s, a), r_{t}\right)$ where

1) $T=0, \ldots, N$ are the decision time steps at which the decisions are made ${ }^{2}$

2) $S$ is a finite set of states;

3) $A(s)$ is a finite set of actions that the agent can select in a state $s \in S$;

\footnotetext{
${ }^{1}$ Several equivalent formulas are available to compute the Stirling number of the second kind, in this case we use $S(n, k)=\frac{1}{k !} \sum_{l=0}^{k}(-1)^{l}\left(\begin{array}{c}k \\ l\end{array}\right)(k-l)^{n}$.

${ }^{2}$ Time steps can be days, hours, minutes or any time interval;
} 
4) $P_{t}\left(s^{\prime} \mid s, a\right)$ is the state transition probability distribution, it encodes the probability at time $t$ of going to state $s^{\prime}$ when the agent is in state $s$ and executes action $a$;

5) $r_{t}: S \times A \longrightarrow \mathbb{R}$ is the Reward Function, $r_{t}(s, a)$ quantifies the value gained when executing action $a$ in state $s$ at time step $t$.

Definition 2: A decision rule $D_{t}$ is a function depending on time step $t$ that defines what action $D_{t}(s) \in A(s)$ at time $t$ the agent should select. By assuming $N$ number of time steps, we define policy $\pi=\left(D_{1}, \ldots, D_{N-1}\right)$ as a sequence of $N-1$ decision rules.

A policy is stationary if the decision rule for all time steps are the same, i.e.,: $\forall t \in\{1, \ldots, T\} D_{t}=D$. A solution for an MDP is a policy $\pi: S \longrightarrow A$ that associates an action to each state. Normally, policies are evaluated by a value function $v^{\pi}: S \longrightarrow \mathbb{R}$. The value function is computed recursively as follows:

$$
v_{N}^{\pi}(s)=r_{N}(s, \pi(s)) \forall s \in S_{T}
$$

where $S_{T}$ is the set of terminal states as a subset of all states. For the rest of time steps $t<T$, the value function is defined as

$$
v_{t}^{\pi}(s)=r_{t}(s, \pi(s))+\gamma \sum_{s^{\prime} \in S} P_{t}\left(s^{\prime} \mid s, \pi(s)\right) v_{t+1}^{\pi}\left(s^{\prime}\right)
$$

where $\gamma$ is a discount factor $(0<\gamma \leq 1) \cdot v_{t}^{\pi}(s)$ represents the expected gain in the future from state $s$, at time step $t$ if the policy $\pi$ is executed. Therefore, the final value of a policy is given by $v^{\pi}=v_{0}^{\pi}$. Let $\pi$ and $\pi^{\prime}$ be two policies, in the following, we indicate $\pi \succeq \pi^{\prime}$ if the policy $\pi$ is better than policy $\pi^{\prime}$

$$
\pi \succeq \pi^{\prime} \Leftrightarrow \forall s \in S v^{\pi}(s) \geq v^{\pi^{\prime}}(s) .
$$

Equation (6) indicates that policy $\pi$ is better than policy $\pi^{\prime}$ if its value is higher than the value of $\pi^{\prime}$ for all states. $\pi^{*}$ is an optimal policy if $\pi^{*} \succeq \pi$, for all feasible policies $\pi$.

What remains to show is how to find an optimal policy. To find the value of the optimal policy, we can use a dynamic programming, namely, the Bellman equation [27]

$$
v_{t}^{*}(s)=\max _{a \in A(s)}\left\{r_{t}(s, a)+\gamma \sum_{s^{\prime} \in S} P_{t}\left(s^{\prime} \mid s, a\right) v_{t+1}^{*}\left(s^{\prime}\right)\right\} .
$$

For extracting the optimal policy, we need to define a $Q$-value function on state $s$ and action $a$ at time step $t$ as

$$
Q_{t}(s, a)=r_{t}(s, a)+\gamma \sum_{s^{\prime} \in S} P_{t}\left(s^{\prime} \mid s, a\right) v_{t+1}^{*}\left(s^{\prime}\right) .
$$

The optimal policy selects action $a^{*}$ at state $s$ and stage $t$ as follows:

$$
a_{t}^{*} \in \underset{a \in A(s)}{\operatorname{argmax}}\left\{Q_{t}(s, a)\right\} \text { for } t=1 \ldots N-1 .
$$

In the following, we present a generalization of MDPs where the rewards are vectors instead of scalars.

Definition 3 (see [40]): A discrete-time VMDP is defined by a tuple $\left(T, S, A, P_{t}(. \mid s, a), \bar{r}_{t}\right)$ where $T, S, A, P_{t}(. \mid s, a)$ are the same as in Definition 1.
1) $\bar{r}_{t}: S \times A \longrightarrow \mathbb{R}^{d}$ is a vector-valued reward function defined as $\bar{r}_{t}(s, a)=\left(r_{1 t}(s, a), \ldots, r_{d t}(s, a)\right)$

In the following section, we will see that VMDPs are particularly suited for services composition context, because each executed service has several quality values, such as response time, throughput, price, availability, etc.

\section{B. Services Composition as a Discrete-Time VMDP}

In this section, we adapt VMDPs in the context of services composition problems. The solution of such MDPs gives the optimal service composition. There are basically two reasons for using an MDP.

1) MDPs make it easy and natural to model services composition problems: Every salient aspect present in the decision-making process relating to the services composition problem has an evident counterpart in an MDP context. The division of an MDP into states and actions is particularly suitable for representing the division of a composition service problem into abstract services and concrete services. Furthermore, an optimal policy in an MDP reduces to choose which actions to take at each state, while the optimal workflow reduces to find the best concrete service for each abstract service.

2) MDPs can be easily integrated into a dynamic environment with uncertainty. In this article, we use part of the results presented in [2] and [41] on how to efficiently learn the users' preferences. These results are based on the combined use of MDP and RL techniques.

From now on, to be coherent with the services composition terminology, we will use the term workflow as a synonym of policy, abstract service as a synonym of state, concrete service as a synonym of action, and QoS as a reward value.

Definition 4: A concrete service $S_{i j}$ can be described by several functional and nonfunctional properties.

1) Functional properties: Each functional property is described through a transaction function, $\operatorname{Action}\left(S_{i j}\right)$ that takes an input data vector InputData $\left(S_{i j}\right)$ to produce an output data vector OutputData $\left(S_{i j}\right)$.

2) Nonfunctional properties: Each nonfunctional property consists of a vector of QoS attributes $Q\left(S_{i j}\right)$, a set of quality of experience criteria, and other aspects about the service such as energy consumption and the context of use.

Definition 5: An abstract service $S_{i}=\left\{S_{i 1}, \ldots, S_{i n_{i}}\right\}$ is a class of $n_{i}$ concrete services with similar functional properties, i.e., they have the same input and output data vectors, but their nonfunctional properties can be different.

In the rest of this section, we will explain how various classes of abstract services, each one associated to several concretes services, can be modeled as a VMDP.

Definition 6: A VMDP-Service Composition (VMDP-SC) is defined by a tuple $\left(T, A S, C S, P_{t}(. \mid a s, c s), \bar{r}_{t}\right)$ similar to Definitions 1 and 3 given as follows:

1) $T=0, \ldots, N$ are the decision time steps;

2) AS is a finite set of abstract services; 
3) $\operatorname{CS}\left(S_{i}\right)$ is a finite set of executable concrete services that the agent can use for a chosen abstract service $S_{i} \in \mathrm{AS}$. We have that $\mathrm{CS}\left(S_{i}\right)=\left\{S_{i 1}, \ldots, S_{i n_{i}}\right\}$;

4) $P_{t}\left(S_{j} \mid S_{i}, S_{i k}\right)$ is the probability of executing concrete service $S_{i k}$ for abstract service $S_{i}$ and entering to abstract service $S_{j}$;

5) $\overline{\mathrm{QoS}}_{t}: \mathrm{AS} \times \mathrm{CS} \longrightarrow \mathbb{R}^{d}$ is the vector valued reward function. $\overline{\mathrm{QoS}}_{t}\left(S_{i}, S_{i k}\right)$ represents a vector of QoS attribute values after invoking $S_{i k}$ in $S_{i}$ at time step $t$. For $d$ attributes, we obtain $\overline{\mathrm{QoS}}_{t}\left(S_{i}, S_{i k}\right)=$ $\left(\operatorname{qos}_{1 t}\left(S_{i}, S_{i k}\right), \ldots, \operatorname{qos}_{d t}\left(S_{i}, S_{i k}\right)\right)$.

The terminal states, defined as $\mathrm{AS}_{T}$, do not have any executable services.

Definition 7: A workflow $\pi: \mathrm{AS} \longrightarrow \mathrm{CS}$ is a function that defines, which concrete service should be invoked for each abstract service in order to give the best tradeoff among multiple QoS attributes.

Since the rewards used in an MDP-SC are vectorial, also the value function $\bar{v}$ and the $Q$-value function $\bar{Q}$ are vectorial

$$
\begin{aligned}
\bar{v}_{t}^{\pi}\left(S_{i}\right)= & \overline{Q o S}_{t}\left(S_{i}, \pi\left(S_{i}\right)\right) \\
& +\gamma \sum_{S_{j} \in A S} P_{t}\left(S_{j} \mid \pi\left(S_{i}\right), S_{i}\right) \bar{v}_{t+1}^{\pi}\left(S_{j}\right) .
\end{aligned}
$$

Thus, comparing two workflows boils down to comparing two vectors. The optimal workflow can differ from user to user according to their preference with respect to the $d$ value qualities of services. We make the assumption that each user assign a different weight $\bar{w}_{i}$ to each QoS attribute. The simple additive weighting (SAW) technique [28] is used to obtain a single QoS value as a weighted linear combination of the QoS attributes. Let $\bar{W}=\left(w_{0}, \ldots, w_{d}\right)$ be the set of normalized weights (i.e., $\sum_{i=1}^{d} w_{i}=1$ ) given by a user, the aggregated QoS becomes

$$
\operatorname{QoS}_{t}\left(S_{i}, S_{i j}\right)=\bar{W} \cdot \overline{\operatorname{QoS}}\left(S_{i}, S_{i j}\right)=\sum_{k=1}^{d} w_{k} \mathrm{qos}_{k t} .
$$

If the user preferences on QoS attributes are given, the optimal workflow can be, therefore, computed using SAW techniques. However, determining appropriate weights for QoS attributes requires the knowledge of the user preferences, which is often not obvious to obtain in practice. Even if the user preferences have been obtained, setting accurately these weights remains a problem. For instance, it is hard to decide the weight of an attribute such as response time as 0.2 or 0.21 , which appears no big difference yet it can affect the result of the QoS optimal composition [10]. We assume that $\bar{W}$ is unknown, and we try to find the best workflow by querying the users when it is necessary.

The basic idea is to deduce the user weights by comparison: we present to the users two alternative QoS vectors, and we assume that he/she is always able to find which of the two he/she prefers. In order to compute the optimal workflow in the service composition, it is required to compare the vector value function of two different workflows.

To compare workflow vector values with each other, we consider first the $d-1$-dimensional polytope $\mathcal{W}$ that represents the set of all possible values for $\bar{W}$

$$
\mathcal{W}=\left\{\left(w_{1}, \ldots, w_{d}\right) \in \mathbb{R}_{+}^{d} \mid \sum_{i=2}^{d} w_{i} \leq 1, w_{1}=1-\sum_{i=2}^{d} w_{i}\right\} .
$$

Three methods can be used to compare vector values associated to the workflows [41]. Assume $\bar{v}^{\pi_{a}}=\left(a_{1}, \ldots, a_{d}\right)$ and $\bar{v}^{\pi_{b}}=\left(b_{1}, \ldots, b_{d}\right)$ are two vector values associated to two workflows $\pi_{a}$ and $\pi_{b}$ given as follows.

1) Pareto comparison:

$$
\bar{v}^{\pi_{a}} \succeq_{P} \bar{v}^{\pi_{b}} \Leftrightarrow \quad \forall i a_{i} \geq b_{i} .
$$

2) K-dominance comparison: $\bar{v}^{\pi_{a}}$ is preferred than $\bar{v}^{\pi_{b}}$ if it is better for any $\bar{w}$ in the polytope $\mathcal{W}$

$$
\bar{v}^{\pi_{a}} \succeq_{K} \bar{v}^{\pi_{b}} \Leftrightarrow \quad \forall \bar{W} \in \mathcal{W}, \bar{W} \cdot \bar{v}^{\pi_{a}} \geq \bar{W} \cdot \bar{v}^{\pi_{b}} .
$$

3) Query to the user: $\bar{v}^{\pi_{a}} \succeq_{q} \bar{v}^{\pi_{b}}$ if the user prefers workflow $\bar{v}^{\pi_{a}}$ over $\bar{v}^{\pi_{b}}$. This check is a sort of "last resort": if none of the previous methods allow to eliminate one of the two vectors, the only option is to ask directly to the user.

It is possible to show that checking whether a K-dominance exists between two workflow vector values can be done by solving an ad hoc linear programming problem [41]: $\bar{v}^{\pi_{a}} \succeq_{K} \bar{v}^{\pi_{b}}$ is true if there is a non-negative solution to the following linear program:

$$
\begin{aligned}
& \min \bar{W} \cdot\left(\bar{v}^{\pi_{a}}-\bar{v}^{\pi_{b}}\right) \\
& \text { s.t. } \bar{W} \in \mathcal{W} .
\end{aligned}
$$

We noticed that

$$
\bar{v}^{\pi_{a}} \succeq_{K} \bar{v}^{\pi_{b}} \not \bar{v}^{\pi_{b}} \succeq_{K} \bar{v}^{\pi_{a}}
$$

therefore, to check whether a dominance exists between the two vectors, we need to solve two separate linear programs. In the remaining of this article, we explain how to find the optimal workflow that gives the best tradeoff among multiple QoS criteria, satisfying the user requirements in terms of QoS only querying a few times.

\section{InTERACTIVE RL AlgORITHMS FOR THE SERVICES COMPOSITION PROBLEM}

After modeling the services composition environment as a VMDP-SC, we are interested in finding a solution for our model, i.e., how to compute the optimal workflow respecting the users' preferences on the QoS attributes. In this section, we introduce an algorithm with this aim, namely, interactive value iteration for services composition (IVI-SC). It is possible to use interactive value iteration methods for finding the optimal workflow by learning user's preferences weights dynamically [2], [41].

We assume that a VMDP-SC with finite discrete-time is given. The services can be invoked in $T+1$ number of discrete time steps: $\{0, \ldots, T-1\} \cup\{T\}$ where $T$ is a final empty time step. Since the MDP-SC objective is finding the workflow that maximizes a measure of long-run expected $\bar{Q}$ vector values, we propose a backward induction method to solve the Bellman 

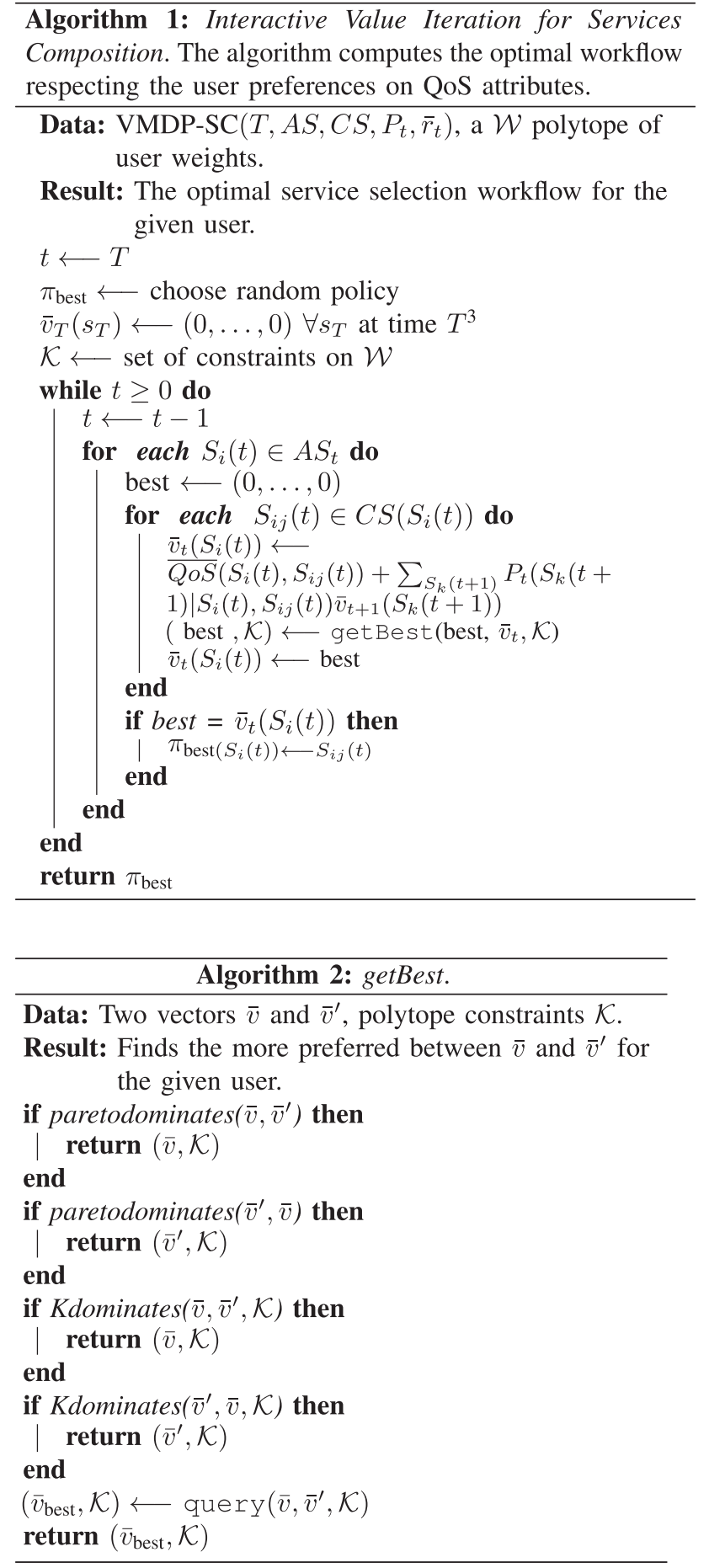

equation given in (7) to obtain the optimal policy/workflow, see (9). Our approach is formalized in Algorithm 1.

In the iterative Algorithm 1, first, we assign a zero vector to the set of states (abstract services) at time step $T$. For each abstract service $S_{i}(t)$ (at time $t$ ) given in the MDP-SC, the algorithm selects the best concrete service among the all available ones. These actions (concrete services) are dependent on various time steps, for instance, the possible actions of the $S_{i}(t)$ service at times step $t$ can be different from the possible actions for time step $t+1$. In the finite horizon time (our case), the iteration

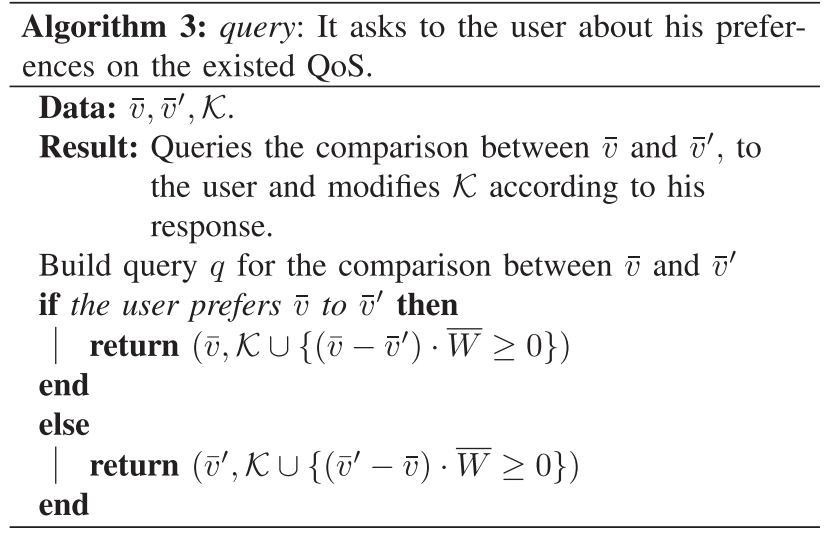

continues until either this difference becomes small enough or the horizon time steps finish.

Since the QoSs is the $d$-dimensional vectors, solving (7) and finding the maximum among the vectors is not obvious. For this reason, we use the Algorithm 2 getBest that applies the three comparison methods presented at the end of Section IV. The getBest function receives two $d$-dimensional vectors with the $\mathcal{W}$ polytope constraining the user weight preferences on the QoSs. If the Pareto comparison cannot find the greater vector, the K-dominance comparison is used to find the most preferred vectors. Otherwise, the query function should be called (given in Algorithm 3). The user's response to the comparison between the two given vectors, adds a new constraint to the $\mathcal{W}$ polytope.

Algorithm 1 finally finds the optimal policy/workflow or services composition for the given system VMDP-SC and returns back the optimal policy $\pi_{\text {best }}$. Notice that the condition best $=\bar{v}_{t}\left(S_{i}(t)\right)$ in Algorithm 1 checks if the best selected concrete service for $S_{i}(t)$ has been changed regarding the previous iteration. If it was, the optimal concrete service should be replaced by the concrete service $S_{i j}(t)$ which generates a better vector value for $S_{i}(t)$.

The proposed algorithm is exact, and its complexity is polynomial w.r.t three parameters: the number of abstracts services forming the composition, the number of candidates concretes services per each abstract services, and the number of QoS attributes. Assuming $|\mathrm{AS}|$ is the set of all abstract services and $M=\max _{i, t} \operatorname{CS}\left(S_{i}(t)\right)$ is the maximum number of abstract services in each time step $t$ and each abstract service $S_{i}$. In order to compute the best QoS vector in each inner iteration, the Best algorithm tests the Pareto dominance and K-dominance comparison twice in the worst case, which are polynomial w.r.t $d$, where $d$ is the number of attributes for QoSs and any K-dominance (LP) can be solved in polynomial time. Finally, we suppose that the time necessary to ask a preference to the user is constant. Therefore, the complexity of the algorithm is $O(M d|A S|)$.

\section{PERformance EVAluation}

We evaluate our methods on a publicly available dataset containing two QoS attributes: throughput and response time. These are the records between 339 users and 5825 web services 


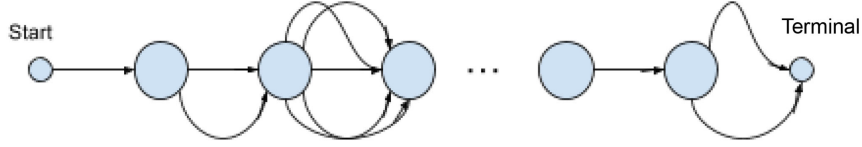

Fig. 3. Sequential form of abstract service connection. The start state is an empty state and it is connected to the first selected abstract service in the model. While the terminal state is an empty state to indicate that we have a finite horizon VMDP-SC.

distributed worldwide [49], [50]. The dataset also includes some information about user features and service features such as countries, autonomous systems, IP dresses, latitude, and longitude. In the studied database, 142 users execute various web services in different time steps. The information is available only for 64 time steps. In this section, we first explain how to model the dataset as a VMDP-SC and then we examine the performances of our algorithm on the dataset.

\section{A. Dataset as a VMDP-SC}

The main issue in implementing IVI-SC (Algorithm 1) on any database is how to model the given dataset as a VMDP. In the supported dataset [49], [50], there are several text files, including wslist.txt, userlist.txt, rtdata.txt, and tpdata.txt. The wslist.txt is our source file for extracting a list of web services and their related abstract services. In our dataset, we consider the web services as the concrete services. The userlist.txt file includes some information about users of different web services. The two other tpdata.txt and rtdata.txt files include the throughput and response time values, respectively, on various web services executed by 142 users. This means that any web service invoked by a user has two parameters for measuring the service quality: throughput (megabits per second, Mbps) and response time (second, s).

The studied database [49], [50] is generated in practice by observing various users utilizing enormous number of web services. After cleaning the database and extracting all web services and their related abstract services from the wslist.tx file, and getting the web services qualities from two files tp.txt and rt.txt, we have a VMDP-SC with the following parameters (see Definition 6):

1) 64 time steps;

2) 744 abstract services;

3) 3551 total number of concrete services (in our case web services);

4) The transition function is not given directly in the database. Several models can be used to define the possible orders and concurrences among the abstract services (see Section III. In this case, we adopt a sequential structure (see Fig. 3);

5) The $\overline{\mathrm{QoS}}_{t}$ function comes from the extracted data on web services and their two qualities: response time and throughput.

To demonstrate the efficiency of our approach in calculating the optimal workflow, we study our method on a common stateof-the-art model: the sequential model.
TABLE II

EXAMPLE OF Two-Dimensional WeIGHT VeCtors (THROUGHPUT AND RESPONSE TIME) FOR FIVE USERS WITH PREFERENCES ON THE ATTRIBUTES

\begin{tabular}{cc}
\hline user & weight vector \\
\hline & [throughput, response time] \\
\hline $\bar{W}_{0}$ & {$[0.320,0.680]$} \\
$\bar{W}_{1}$ & {$[0.857,0.143]$} \\
$\bar{W}_{2}$ & {$[0.170,0.830]$} \\
$\bar{W}_{3}$ & {$[0.645,0.355]$} \\
$\bar{W}_{4}$ & {$[0.472,0.528]$} \\
\hline
\end{tabular}

The goal of Algorithm 1 is to learn such a vectors for each of the five users.

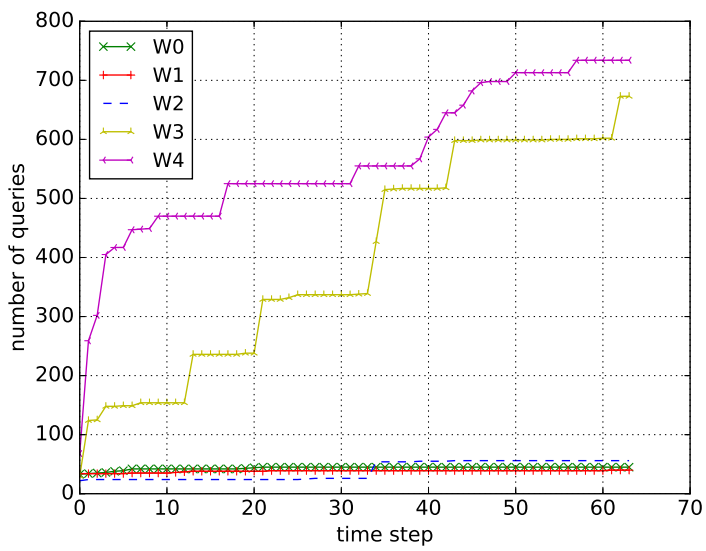

Fig. 4. This figure shows the number of queries proposed to the user during each time step. The weight preferences are based on Table II.

For the sequential model (see Fig. 3), the start state is an empty state connected to the first selected abstract service in the model. On the contrary, the terminal state is an empty state that indicates that the MDP has a finite horizon. The sequential order on abstract services can be defined in any order. In our model, the order is randomly selected once, to fix the MDP model. For any time step $t \in\{0 \ldots 63\}$, the transition probability $P_{t}\left(S_{j}(t+1) \mid S_{i}(t), S_{i k}(t)\right)$ is 1 , if web service $S_{i k}$ is invocable for a given abstract service $S_{i}(t)$ according to our database and the abstract service $S_{j}(t+1)$ is the next demanded service in our selected sequential MDP model, otherwise $P_{t}\left(S_{j}(t+1) \mid S_{i}(t), S_{i k}(t)\right)=0$.

\section{B. Model the Web Service Dataset as VMDP}

To evaluate Algorithm 1, we consider the complete dataset, i.e., we keep all the quality services executed by all 142 users. Modeling such a huge size database as a VMDP-SC and implementing the IVI-SC algorithm on it is a challenging task.

In order to evaluate our algorithm performance, we analyze the results for five different users with various preferences on the service qualities (response time and throughput). Our tested user weights vectors $(\bar{W})$ on service qualities are given in Table II. Notice that the weight preferences on the QoSs are "unknown" to our algorithm. They are used to simulate the user's behavior.

Fig. 4 shows how the interactive value iteration algorithm communicates with users during the 64 time steps. Since the user weight preferences are unknown to the algorithm, it is 


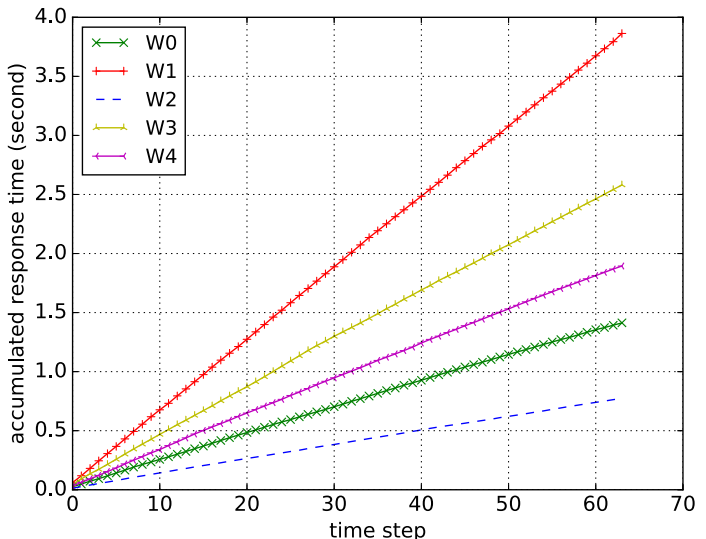

Fig. 5. Figure shows how the accumulated response time increases during each time step. The weight preferences are based on Table II.



Fig. 6. Figure shows how the accumulated throughput increases during each time step. The weight preferences are based on Table II.

needed to query them in the required situations. According to the figure, Algorithm 1 finds the optimal workflows after asking less than 56 queries to the users with preferences weights $\bar{W}_{0}$, $\bar{W}_{1}$, and $\bar{W}_{2}$. Contrarily, for the users with weight preferences $\bar{W}_{3}$ and $\bar{W}_{4}$, the algorithm queries a more significant amount of questions to the user. One possible explanation for the different behavior from one user to the other is that, if the weights are more balanced, comparing vectors using Pareto dominant and $\mathrm{K}$-dominant methods is less informative and, therefore, the only option to eliminate one of the two vectors is to ask directly to the user. This problem can be mitigated by approximating a workflow for the system instead of calculating the exact one. Since IVI-SC is an exact algorithm, the initial sequence of dataset presentation have an important effect on the number of required queries in order to find the optimal workflow.

Figs. 5 and 6 shows how the service qualities change with respect to the time step for the five given weight preferences. The optimal workflow should maximize the total sum of throughputs while minimizing the total sum of response times. The two figures show how throughput and response time increase linearly w.r.t the time steps. Fig. 5 shows that the accumulated response
TABLE III

It Demonstrates How the COMPUTED Workflow From ALgorithm 1 Is DIFFERENT FROM THE IDEAL WORKFLOW FOR $\overline{W_{3}}$

\begin{tabular}{|c|c|c|c|c|c|c|c|}
\hline Services & $\begin{array}{l}\begin{array}{c}\text { number } \\
\text { of WS }\end{array} \\
\text {. }\end{array}$ & IVI & Exact & Services & $\begin{array}{l}\begin{array}{c}\text { number } \\
\text { of WS }\end{array} \\
\end{array}$ & IVI & Exact \\
\hline AS14280 & 2 & 566 & 567 & AS9829 & 2 & 1559 & 1565 \\
\hline AS6360 & 3 & 3723 & 3725 & AS29650 & 2 & 1583 & 1586 \\
\hline AS4766 & 9 & 2226 & 2237 & AS4768 & 2 & 2053 & 2054 \\
\hline AS 3288 & 2 & 1622 & 1623 & AS42695 & 2 & 2503 & 2504 \\
\hline AS20284 & 8 & 1547 & 1554 & AS42915 & 3 & 1588 & 1590 \\
\hline AS852 & 7 & 533 & 4126 & AS22047 & 2 & 612 & 613 \\
\hline AS14415 & 13 & 4120 & 4119 & AS50517 & 3 & 2304 & 2306 \\
\hline AS 16265 & 2 & 2018 & 2665 & AS5050 & 4 & 3584 & 3586 \\
\hline AS13301 & 6 & 1382 & 1487 & AS13041 & 24 & 2375 & 2368 \\
\hline AS15806 & 7 & 1566 & 1576 & AS 47720 & 3 & 1569 & 1571 \\
\hline AS11955 & 12 & 3882 & 3888 & AS 8075 & 11 & 12 & 4183 \\
\hline AS33821 & 2 & 2256 & 2258 & AS23148 & 5 & 583 & 4233 \\
\hline AS29944 & 3 & 4223 & 4224 & AS35041 & 2 & 2514 & 2533 \\
\hline AS20773 & 6 & 1348 & 2548 & AS6830 & 2 & 1579 & 1584 \\
\hline AS17819 & 4 & 2325 & 2329 & AS7050 & 4 & 4198 & 4201 \\
\hline AS27437 & 3 & 4047 & 4050 & AS48347 & 3 & 2271 & 2273 \\
\hline AS19855 & 3 & 3652 & 3653 & AS760 & 3 & 90 & 92 \\
\hline AS23650 & 22 & 625 & 853 & AS8737 & 13 & 1932 & 1940 \\
\hline AS87 & 5 & 4111 & 4112 & AS9116 & 2 & 1615 & 1630 \\
\hline AS8551 & 4 & 1620 & 1634 & AS19875 & 5 & 547 & 549 \\
\hline AS15366 & 4 & 1244 & 1246 & AS55481 & 4 & 44 & 47 \\
\hline AS5603 & 2 & 2345 & 2349 & AS39418 & 10 & 959 & 970 \\
\hline AS31727 & 4 & 1582 & 2922 & AS5786 & 3 & 2212 & 2214 \\
\hline AS9848 & 4 & 2232 & 2233 & AS29097 & 3 & 2538 & 2540 \\
\hline AS29076 & 5 & 2275 & 2293 & AS29951 & 8 & 3856 & 3860 \\
\hline AS4808 & 10 & 670 & 770 & AS32577 & 10 & 3640 & 3638 \\
\hline AS17431 & 2 & 752 & 753 & AS32475 & 3 & 3728 & 3729 \\
\hline AS2819 & 5 & 918 & 946 & AS3786 & 13 & 2215 & 2225 \\
\hline AS32613 & 3 & 93 & 94 & AS27030 & 3 & 4467 & 4469 \\
\hline AS11305 & 3 & 3688 & 4046 & AS24969 & 2 & 999 & 1004 \\
\hline AS15670 & 3 & 1982 & 1984 & AS1251 & 10 & 175 & 194 \\
\hline AS156 & 2 & 4179 & 4180 & AS12714 & 2 & 2279 & 2280 \\
\hline AS16095 & 3 & 998 & 1016 & AS16245 & 2 & 1000 & 1018 \\
\hline AS15290 & 6 & 536 & 574 & AS8542 & 6 & 2081 & 2090 \\
\hline AS31815 & 11 & 4236 & 4414 & AS34235 & 4 & 1141 & 1208 \\
\hline AS8151 & 3 & 1918 & 1919 & AS9308 & 5 & 748 & 801 \\
\hline AS4812 & 11 & 760 & 862 & AS5409 & 2 & 1440 & 1441 \\
\hline AS3389 & 4 & 4109 & 4106 & AS8928 & 2 & 1202 & 3045 \\
\hline AS81 & 5 & 509 & 510 & AS3561 & 25 & 3647 & 3579 \\
\hline AS6785 & 2 & 955 & 956 & AS8659 & 4 & 2543 & 2545 \\
\hline AS45061 & 12 & 648 & 649 & AS44249 & 10 & 2171 & 2173 \\
\hline AS702 & 16 & 156 & 1147 & AS701 & 19 & 3493 & 3491 \\
\hline AS22070 & 2 & 3661 & 3662 & AS11426 & 50 & 3207 & 3163 \\
\hline AS1128 & 5 & 1964 & 1965 & AS2611 & 10 & 132 & 140 \\
\hline AS25518 & 2 & 1774 & 1775 & AS14584 & 4 & 3894 & 3896 \\
\hline AS224 & 7 & 2115 & 2075 & AS16339 & 2 & 2911 & 2912 \\
\hline AS15348 & 3 & 3731 & 3732 & AS16237 & 4 & 1959 & 1960 \\
\hline AS31827 & 3 & 4341 & 4342 & AS32 & 9 & 4387 & 4388 \\
\hline AS30190 & 2 & 4408 & 4409 & AS9143 & 8 & 1974 & 1991 \\
\hline AS8220 & 15 & 160 & 1182 & AS9318 & 6 & 2229 & 2243 \\
\hline AS4230 & 8 & 167 & 199 & AS22489 & 3 & 210 & 211 \\
\hline AS43200 & 3 & 2087 & 2089 & AS3307 & 3 & 2091 & 2120 \\
\hline AS3301 & 6 & 2506 & 2522 & AS9811 & 3 & 695 & 697 \\
\hline AS34779 & 9 & 2332 & 2335 & AS73 & 4 & 3911 & 3914 \\
\hline AS553 & 2 & 1448 & 1466 & AS12859 & 7 & 125 & 2024 \\
\hline AS 15756 & 3 & 2283 & 2298 & AS15879 & 4 & 1961 & 2029 \\
\hline AS10929 & 4 & 4065 & 4066 & AS15085 & 2 & 554 & 555 \\
\hline AS21844 & 13 & 4163 & 4161 & AS12731 & 4 & 1361 & 1378 \\
\hline AS237 & 6 & 3523 & 816 & AS195 & 13 & 4188 & 4027 \\
\hline AS1226 & 3 & 4010 & 4012 & AS1221 & 2 & 43 & 79 \\
\hline AS 8358 & 2 & 1521 & 1522 & AS680 & 44 & 1398 & 1401 \\
\hline AS8 & 3 & 3667 & 3668 & AS3316 & 4 & 2260 & 2261 \\
\hline AS4837 & 29 & 680 & 791 & AS27617 & 3 & 4445 & 4446 \\
\hline AS8190 & 3 & 4067 & 4068 & AS8904 & 5 & 2264 & 2266 \\
\hline AS43541 & 3 & 913 & 915 & S2519 & 4 & 1857 & 1859 \\
\hline AS42949 & 2 & 1994 & 1995 & AS18125 & 5 & 1861 & 1862 \\
\hline AS2200 & 28 & 1120 & 1108 & AS26228 & 6 & 2077 & 2080 \\
\hline AS19024 & 8 & 3924 & 3929 & AS11343 & 3 & 4444 & 4442 \\
\hline AS40142 & 7 & 3740 & 3767 & AS1103 & 3 & 1989 & 1992 \\
\hline AS33491 & 3 & 3681 & 3682 & AS33494 & 9 & 3700 & 3707 \\
\hline AS12695 & 3 & 2277 & 2291 & AS9120 & 2 & 1011 & 1028 \\
\hline AS41635 & 3 & 2250 & 2252 & AS21309 & 3 & 1792 & 1794 \\
\hline AS8342 & 5 & 950 & 2269 & AS12306 & 9 & 736 & 4317 \\
\hline AS12301 & 3 & 1512 & 1514 & AS9338 & 9 & 2040 & 2046 \\
\hline AS10694 & 9 & 4123 & 4354 & AS3778 & 7 & 3839 & 3838 \\
\hline AS6983 & 50 & 3214 & 3234 & AS43220 & 2 & 980 & 981 \\
\hline AS39111 & 3 & 964 & 1319 & AS3614 & 4 & 3539 & 3541 \\
\hline AS17477 & 9 & 18 & 2062 & AS29134 & 2 & 939 & 940 \\
\hline AS43892 & 2 & 1532 & 1533 & AS14335 & 2 & 3596 & 3597 \\
\hline S8972 & 3 & 1343 & 1344 & AS3292 & 36 & 2068 & 2478 \\
\hline AS8803 & 3 & 2554 & 2555 & AS12874 & 23 & 1711 & 1821 \\
\hline AS1930 & 2 & 2204 & 2208 & AS210 & 13 & 3709 & 3712 \\
\hline S24958 & 3 & 947 & 948 & AS15083 & 28 & 863 & 864 \\
\hline AS6400 & 4 & 1030 & 1031 & AS1205 & 4 & 108 & 111 \\
\hline AS8508 & 3 & 2168 & 2169 & AS11798 & 18 & 3822 & 3824 \\
\hline S36017 & 3 & 4473 & 4474 & AS55454 & 3 & 2031 & 2032 \\
\hline AS36850 & 4 & 3655 & 3657 & AS 40619 & 4 & 3526 & 3525 \\
\hline AS16276 & 8 & 1191 & 1201 & AS5537 & 2 & 2288 & 2295 \\
\hline AS13367 & 10 & 3750 & 3738 & AS 12312 & 4 & 1365 & 1367 \\
\hline
\end{tabular}


TABLE IV

It DEMONSTRATES How the COMPUTED WORKFLOW From ALGORITHM 1 IS DIFFERENT FROM THE IDEAL WORKFLOW FOR $\overline{W_{4}}$

\begin{tabular}{lc|cc||cc||cc}
\hline Services & $\begin{array}{c}\text { number } \\
\text { of ws }\end{array}$ & IVI & Exact & Services & $\begin{array}{c}\text { number } \\
\text { of ws }\end{array}$ & IVI & Exact \\
\hline AS42695 & 2 & 2503 & 2504 & AS50517 & 3 & 2304 & 2306 \\
AS16265 & 2 & 2018 & 2665 & AS15806 & 7 & 1566 & 1576 \\
AS8075 & 11 & 3830 & 4183 & AS23148 & 5 & 583 & 4233 \\
AS35041 & 2 & 2514 & 2533 & AS48347 & 3 & 2271 & 2273 \\
AS760 & 3 & 90 & 92 & AS39418 & 10 & 963 & 970 \\
AS4808 & 10 & 670 & 770 & AS32475 & 3 & 3728 & 3729 \\
AS32613 & 13 & 99 & 94 & AS156 & 2 & 4179 & 4180 \\
AS25260 & 5 & 1268 & 1270 & AS16245 & 2 & 1000 & 1018 \\
AS8542 & 6 & 2081 & 2090 & AS5409 & 2 & 1440 & 1441 \\
AS3561 & 25 & 3647 & 3579 & AS2611 & 10 & 132 & 135 \\
AS14584 & 4 & 3894 & 3896 & AS16237 & 4 & 1959 & 1960 \\
AS10929 & 4 & 4065 & 4066 & AS12731 & 4 & 1361 & 1378 \\
AS4837 & 29 & 739 & 791 & AS2519 & 4 & 1857 & 1859 \\
AS18125 & 5 & 1861 & 1862 & AS2200 & 28 & 1120 & 1052 \\
AS26228 & 6 & 2077 & 2080 & AS41635 & 3 & 2250 & 2252 \\
AS21309 & 3 & 1792 & 1794 & AS10694 & 9 & 4123 & 4124 \\
AS3778 & 7 & 3836 & 3838 & AS39111 & 3 & 964 & 1319 \\
AS3614 & 4 & 3539 & 3541 & AS1930 & 2 & 2204 & 2208 \\
AS8508 & 3 & 2168 & 2169 & AS36850 & 4 & 3656 & 3657 \\
\hline
\end{tabular}

time for all weight preferences does not exceed 4 s. On the contrarily, Fig. 6 maximizes the accumulated throughput until a value of around $9000 \mathrm{Mb} / \mathrm{s}$.

If the user's weight vectors are available, the exact workflow is computed by taking the weights into account and using a classical approach on MDPs such as Value iteration or Policy Iteration method [5], [34]. In order to compare the workflows obtained with our method with the ideal ones, we transfer the quality vectors to the quality values: $\operatorname{QoS}\left(S_{i}, S_{i j}\right)=\bar{W} \cdot \overline{\operatorname{QoS}}\left(S_{i}, S_{i j}\right)$ by doing the assumption of knowing a priori the user weights $\bar{W}$. In this way, we can compute the exact services compositions for each user and compare them with the computed workflows from the IVI-SC method. Our experiments confirm that the optimal workflow computed by the IVI-SC algorithm is exactly the same as the exact computed workflow for the three users with weights preferences $\bar{W}_{0}, \bar{W}_{1}$, and $\bar{W}_{2}$. For the two other weight preferences $\bar{W}_{3}$ and $\bar{W}_{4}$, the IVI-SC and the exact approach are different in a few number of abstract services: 178 out of 744 abstract services for $\bar{W}_{3}$ and 38 out of 744 abstract services for $\bar{W}_{4}$. In other words, our method computes $76 \%$ and $95 \%$ of concrete services correctly for users $\bar{W}_{3}$ and $\bar{W}_{4}$, respectively. Table III presents the list of abstract services where our approach (IVI-SC) and the exact approach propose different concrete services for the services composition problem under $\bar{W}_{4}$. Table IV shows the differences between these two approaches for $\bar{W}_{4}$. The first column contains a service abstract name with its possible number of concrete services to execute, and the second column shows the selected concrete service number by the IVI and the exact methods.

\section{CONCLUSION AND FUTURE WORKS}

In this article, an RL-based approach is proposed to solve the services composition problem in a WoT environments and without knowing the user preferences on the QoS attributes. The services composition problem is formulated as a discrete-time VMDP and solved using an interactive value iteration method. The registered qualities of the web services in our studied dataset [49], [50] are executed by selecting 142 users from the dataset. The experiments show that our algorithm finds the optimal services composition by learning the user preferences weights with high accuracy. The optimal services composition is obtained by maximizing the accumulated throughputs and minimizing the accumulated response times. As potential future work, different models of MDPs should be tested, such as parallel MDP, sequential parallel MDP, etc. Finally, it could also be interesting to classify and observe the users types w.r.t. their service qualities and their execution information on the web services before modeling the dataset into an MDP.

\section{REFERENCES}

[1] O. Alagoz, H. Hsu, A. J. Schaefer, and M. S. Roberts, "Markov decision processes: A tool for sequential decision making under uncertainty," Med. Decision Making, vol. 30, no. 4, pp. 474-483, 2009.

[2] P. Alizadeh, Y. Chevaleyre, and F. Lévy, "Advantage based value iteration for Markov decision processes with unknown rewards," in Proc. Int. Joint Conf. Neural Netw., Vancouver, BC, Canada, Jul. 2016, pp. 3837-3844.

[3] M. Alrifai, T. Risse, and W. Nejdl, "A hybrid approach for efficient web service composition with end-to-end QoS constraints," ACM Trans. Web, vol. 6, no. 2, 2012, Art. no. 7.

[4] M. Alrifai, D. Skoutas, and T. Risse, "Selecting skyline services for QoSbased web service composition," in Proc. 19th Int. Conf. World Wide Web, 2010, pp. 11-20.

[5] F. E. M. Arasi, S. Govindarajan, and A. Subbarayan, "Weighted quality of service based ranking of web services," Indian J. Sci. Technol., vol. 9, no. 21, pp. 1-8, Jul. 2017.

[6] J. Cao, J. Huang, G. Wang, and J. Gu, "QoS and preference based web service evaluation approach," in Proc. 8th Int. Conf. Grid Cooperative Comput., 2009, pp. 420-426.

[7] M. Chandra, A. Agrawal, A. Kishor, and R. Niyogi, "Web service selection with global constraints using modified gray wolf optimizer," in Proc. Int. Conf. Adv. Comput., Commun. Informat., 2016, pp. 1989-1994.

[8] N. Chen, N. Cardozo, and S. Clarke, "Goal-driven service composition in mobile and pervasive computing," IEEE Trans. Services Comput., vol. 11, no. 1, pp. 49-62, Jan./Feb. 2018.

[9] X. Chen, Z. Zheng, Q. Yu, and M. R. Lyu, "Web service recommendation via exploiting location and QoS information," IEEE Trans. Parallel Distrib. Syst., vol. 25, no. 7, pp. 1913-1924, Jul. 2014.

[10] Y. Chen, J. Huang, C. Lin, and J. Hu, "A partial selection methodology for efficient QoS-aware service composition," IEEE Trans. Services Comput., vol. 8, no. 3, pp. 384-397, May/Jun. 2015.

[11] A. Chibani, "3 Web of Objects European Project," 2019 (accessed on: Oct. 8, 2019). [Online]. Available: http://www.lissi.fr/woo-en/

[12] S. Deng, L. Huang, W. Tan, and Z. Wu, "Top-k automatic service composition: A parallel method for large-scale service sets," IEEE Trans. Autom. Sci. Eng., vol. 11, no. 3, pp. 891-905, Jul. 2014.

[13] S. Deng, H. Wu, D. Hu, and J. L. Zhao, "Service selection for composition with QoS correlations," IEEE Trans. Services Comput., vol. 9, no. 3, pp. 291-303, Mar. 2016.

[14] A. M. Ejaz, H. Mukhtar, D. Belaid, and J. B. Song, "QoS-aware device selection using user preferences for tasks in ubiquitous environments," in Proc. IEEE Int. Conf. Emerging Technol., 2011, pp. 1-6.

[15] V. Gabrel, M. Manouvrier, and C. Murat, "Web services composition: Complexity and models," Discrete Appl. Math., vol. 196 (Supplement C), pp. 100-114, 2015.

[16] R. L. Graham, D. E. Knuth, and O. Patashnik, Concrete Mathematics, Reading, MA, USA: Addison-Wesley, 1989.

[17] M. Graiet, I. Abbassi, M. Kmimech, and W. Gaaloul, "A genetic-based adaptive approach for reliable and efficient service composition," IEEE Syst. J., vol. 12, no. 2, pp. 1644-1654, Jun. 2018.

[18] M. S. Hossain, M. Moniruzzaman, G. Muhammad, A. Ghoneim, and A. Alamri, "Big data-driven service composition using parallel clustered particle swarm optimization in mobile environment," IEEE Trans. Serv. Comput., vol. 9, no. 1, pp. 806-817, Sep. 2016.

[19] N. M. Josuttis, SOA in Practice: The Art of Distributed System Design. Sebastopol, CA, USA: O’Reilly Media, Inc., 2007.

[20] Y. Lei, Z. Jiantao, W. Fengqi, G. Yongqiang, and Y. Bo, "Web service composition based on reinforcement learning," in Proc. IEEE Int. Conf. Web Services., 2015, pp. 731-734.

[21] J. W. Liu, L. Q. Hu, Z. Q. Cai, L. N. Xing, and X. Tan, "Large-scale and adaptive service composition based on deep reinforcement learning," $J$. Vis. Commun.. Image Representation, vol. 65, 2019, Art. no. 102687. 
[22] W. Liu, "Trustworthy service selection and composition - reducing the entropy of service-oriented web," in Proc. 3rd IEEE Int. Conf. Ind. Informat., Aug. 2005, pp. 104-109.

[23] N. B. Mabrouk, N. Georgantas, and V. Issarny, "Set-based bi-level optimisation for QoS-aware service composition in ubiquitous environments," in Proc. IEEE Int. Conf. Web Services, 2015, pp. 25-32.

[24] D. Mallayya, B. Ramachandran, and S. Viswanathan, "An automatic web service composition framework using qos-based web service ranking algorithm," The Scientific World J., vol. 2015, 2015, Art. no. 207174.

[25] U. S. Manikrao and T. V. Prabhakar, "Dynamic selection of web services with recommendation system," in Proc. Int. Conf. Next Generation Web Serv. Practices, Aug. 2005, p. 5.

[26] A. Mostafa and M. Zhang, "Multi-objective service composition in uncertain environments," IEEE Trans. Serv. Comput., to be published, doi: 10.1109/TSC.2015.2443785.

[27] M. L. Puterman, Markov Decision Processes: Discrete Stochastic Dynamic Programming, 1st ed. New York, NY, USA: Wiley, 1994.

[28] L. Qi, Y. Tang, W. Dou, and J. Chen, "Combining local optimization and enumeration for QoS-aware web service composition," in Proc. IEEE Int. Conf. Web Services, 2010, pp. 34-41.

[29] R. V. Rao, V. J. Savsani, and D. P. Vakharia, "Teaching-learning-based optimization: A novel method for constrained mechanical design optimization problems," Comput.-Aided Des., vol. 43, no. 3, pp. 303-315, 2011.

[30] L. Ren, W. Wang, and H. Xu, "A reinforcement learning method for constraint-satisfied services composition," IEEE Trans. Services Comput., no. 1, Los Alamitos, CA, USA: IEEE Computer Society, Jul. 2017, doi: 10.1109/TSC.2017.2727050.

[31] P. Rodriguez-Mier, C. Pedrinaci, M. Lama, and M. Mucientes, "An integrated semantic web service discovery and composition framework," IEEE Trans. Services Comput., vol. 9, no. 4, pp. 537-550, Jul. 2016.

[32] Q. Z. Sheng, X. Qiao, A. Vasilakos, C. Szabo, S. Bourne, and X. Xu, "Web services composition: A decades overview," Inf. Sciences, vol. 280, pp. 218-238, 2014.

[33] D. Skoutas, D. Sacharidis, A. Simitsis, and T. Sellis, "Ranking and clustering web services using multicriteria dominance relationships," IEEE Trans. Services Comput., vol. 3, no. 3, pp. 163-177, Jul.-Sep. 2010.

[34] R. S. Sutton and A. G. Barto, Reinforcement Learning: An Introduction. Cambridge, MA, USA: MIT Press, 1998.

[35] N. Temglit, A. Chibani, K. Djouani, and M. A. Nacer, "A distributed agentbased approach for optimal QoS selection in web of object choreography," IEEE Syst. J., vol. 12, no. 2, pp. 1655-1666, Jun. 2018.

[36] W. Tsai, P. Zhong, X. Bai, and J. Elston, "Dependence-guided service composition for user-centric SOA," IEEE Syst. J., vol. 8, no. 3, pp. 889899, Sep. 2014.

[37] H. Wang, G. Huang, and Q. Yu, "Automatic hierarchical reinforcement learning for efficient large-scale service composition," in Proc. IEEE Int. Conf. Web Services, 2016, pp. 57-64.
[38] H. Wang, J. Li, Q. Yu, T. Hong, J. Yan, and W. Zhao, "Integrating recurrent neural networks and reinforcement learning for dynamic service composition," Future Generation Comput. Syst., vol. 107, pp. 551-563, 2020.

[39] H. Wang, P. Ma, Q. Yu, D. Yang, J. Li, and H. Fei, "Combining quantitative constraints with qualitative preferences for effective non-functional properties-aware service composition," J. Parallel Distributed Comput., vol. 100, pp. 71-84, 2017.

[40] H. Wang, X. Zhou, X. Zhou, W. Liu, W. Li, and A. Bouguettaya, Adaptive Service Composition Based on Reinforcement Learning. Berlin, Germany: Springer, 2010, pp. 92-107.

[41] P. Weng and B. Zanuttini, "Interactive value iteration for Markov decision processes with unknown rewards," in Proc. 23rd Int. Joint Conf. Artif. Intell., Beijing, China, Aug. 2013, pp. 2415-2421.

[42] Q. Wu, F. Ishikawa, Q. Zhu, and D.-H. Shin, "QoS-aware multigranularity service composition: Modeling and optimization," IEEE Trans. Syst., Man, Cybern.: Syst., vol. 46, no. 11, pp. 1565-1577, Nov. 2016.

[43] P. Xiong, Y. Fan, and M. Zhou, "Web service configuration under multiple quality-of-service attributes," IEEE Trans. Autom. Sci. Eng., vol. 6, no. 2, pp. 311-321, Apr. 2009.

[44] M. E. Khanouche, Y. Amirat, A. Chibani, M. Kerkar, and A. Yachir, "Energy-centered and QoS-aware services selection for Internet of Things," IEEE Trans. Autom. Sci. Eng., vol. 13, no. 3, pp. 1256-1269, Jul. 2016.

[45] H. Yang and X. Xie, "An actor-critic deep reinforcement learning approach for transmission scheduling in cognitive internet of things systems," IEEE Syst. J., vol. 14, no. 1, pp. 51-60, Mar. 2020.

[46] Q. Yu and A. Bouguettaya, "Efficient service skyline computation for composite service selection," IEEE Trans. Knowl. Data Eng., vol. 25, no. 4, pp. 776-789, Apr. 2013.

[47] L. Zeng, B. Benatallah, M. Dumas, J. Kalagnanam, and Q. Z. Sheng, "Quality driven web services composition," in Proc. 12th Int. Conf. World Wide Web, 2003, pp. 411-421.

[48] L. Zeng, B. Benatallah, A. H. H. Ngu, M. Dumas, J. Kalagnanam, and H. Chang, "QoS-aware middleware for web services composition," IEEE Trans. Softw. Eng., vol. 30, no. 5, pp. 311-327, May 2004.

[49] Y. Zhang, G. Cui, S. Deng, and Q. He, "Alliance-aware service composition based on quotient space," in Proc. IEEE Int. Conf. Web Services, Jun. 2016, pp. 340-347.

[50] Z. Zheng, Y. Zhang, and M. R. Lyu, "Investigating QoS of real-world web services," IEEE Trans. Services Comput., vol. 7, no. 1, pp. 32-39, Jan.-Mar. 2014.

[51] G. Zou, Q. Lu, Y. Chen, R. Huang, Y. Xu, and Y. Xiang, "QoS-aware dynamic composition of web services using numerical temporal planning," IEEE Trans. Services Comput., vol. 7, no. 1, pp. 18-31, Jan. 2014. 